\title{
Increased Histone Acetyltransferase and Lysine Acetyltransferase Activity and Biphasic Activation of the ERK/RSK Cascade in Insular Cortex During Novel Taste Learning
}

\author{
Michael W. Swank and J. David Sweatt \\ Division of Neuroscience, Baylor College of Medicine, Houston, Texas 77030
}

\begin{abstract}
Changes in gene expression are thought to be involved in neuronal plasticity associated with learning and memory. Although acetylation of lysine residues on histones by histone acetyltransferases (HAT) is an obligatory component of transcription, HAT activity has been largely ignored in studies of the nervous system. We developed a new model for studying novel taste learning using novel solid food presentation to nondeprived animals. Using this behavioral paradigm, we investigated short- and long-term regulation of lysine acetyltransferase activity and the ERK/mitogen-activated protein kinase (MAPK)/RSK cascade in insular cortex, a CNS region known to be crucial for the formation of novel taste memories. We observed that novel taste learning elicited biphasic (acute and long-lasting) activation of two distinct lysine acetyltransferase
\end{abstract}

The association between histone acetylation and transcriptional regulation has been known for many decades (Allfrey et al., 1964), and it is widely recognized that increased histone acetyltransferase (HAT) activity is an obligatory component of transcription (Davie and Spencer, 1999; Spencer and Davie, 1999; Kouzarides, 2000). Although current models suggest that changes in transcriptional control are necessary for long-term memory formation (Bailey et al., 1996; Impey et al., 1998; Silva et al., 1998; Alberini, 1999; Roberson et al., 1999; Stork and Welzl, 1999), the role of histone acetylation has not been examined in any learning or memory paradigm to date.

Novel taste learning presents several unique attributes that make it a compelling model for looking at gene expression during long-term memory formation: it is robust, long-lasting, and can occur after only a single trial (Bures, 1998). These properties of novel taste learning suggest the hypothesis that altered gene expression may be involved in the formation of the memory.

Consistent with this hypothesis, activation of the ERK/ mitogen-activated protein (MAP) kinase cascade, a prototype cascade for regulating gene expression, has been shown to occur after novel taste experience in the insular cortex, and MAP kinase activation is necessary for novel taste memory formation (Berman et al., 1998). Furthermore, localization of phosphory-

\footnotetext{
Received Dec. 4, 2000; revised Feb. 15, 2001; accepted March 9, 2001.

This research was supported by National Institutes of Health Grants MH18390, DC00454 (M.W.S.), and MH57014 (J.D.S.), a grant from the New York Obesity Research Center (M.W.S.), and a grant from the Texas Advanced Technology Program (J.D.S.). The kind support and encouragement of Dr. Gerard P. Smith are gratefully acknowledged.

Correspondence should be addressed to Michael W. Swank, Division of Neuroscience, Baylor College of Medicine, One Baylor Plaza, Houston, Texas 77030. E-mail:mswank@cns.bcm.tmc.edu.

Copyright (C) 2001 Society for Neuroscience $0270-6474 / 01 / 213383-09 \$ 15.00 / 0$
}

activities along with the ERK/MAPK cascade in insular cortex. In vitro studies revealed that the ERK cascade could regulate the lysine acetylation of a $42 \mathrm{kDa}$ lysine acetyltransferase substrate, suggesting a causal relationship between ERK activation and lysine acetyltransferase activity in insular cortex. Overall, our studies reveal an unanticipated long-lasting activation of insular cortex signal transduction cascades in novel taste learning. Furthermore, our studies suggest the hypothesis that acute and long-term ERK activation and lysine-histone acetyltransferase activation may play a role in regulating gene expression in single-trial learning and long-term memory formation.

Key words: MAP kinase; histone acetyltransferase; protein kinase; taste aversion; insular cortex; learning; memory; gene expression

lated, activated MAP kinase by immunohistochemistry has further defined the insular cortex as being involved in novel taste learning in mice (Swank, 2000a). Although a recent detailed study gave new insight into the neurotransmitters involved in the upstream regulation of MAP kinase by novel taste (Berman et al., 2000), little work has been done on mitogen-activated protein kinase (MAPK)-dependent events that lie downstream of this pathway in insular cortex.

With this in mind, it is interesting that recent evidence suggests that MAP kinase is involved in regulation of histone acetylation and chromatin remodeling through the action of downstream effectors (Thomson et al., 1999). For example, one specific effector is the cAMP response element-binding protein (CREB)binding protein (CBP), an MAPK substrate with intrinsic histone acetyltransferase activity; when phosphorylated by MAP kinase, CBP acetyltransferase activity increases (Ait et al., 1999), resulting in increased transcription (Martinez-Balbas et al., 1998). Therefore, in the present studies, we were interested in evaluating whether histone-lysine acetyltransferase activity is regulated in insular cortex during single-trial novel taste learning. As part of these studies, we also undertook a characterization of the activation of the Raf/MEK/ERK/RSK cascade in insular cortex after single-trial novel taste learning.

\section{MATERIALS AND METHODS}

Mice. Male Swiss Webster mice weighing 30-35 gm were housed individually, received ad libitum food and water, and were maintained on a $12 \mathrm{hr}$ light/dark schedule until training began. All procedures were in accordance with university and National Institutes of Health guidelines.

To test neophobia, mice $(n=3)$ were given a $10 \mathrm{~min}$ access to a preweighed portion of blueberry bar that was then reweighed to measure consumption. On the next day mice were given a second $10 \mathrm{~min}$ access to blueberry bar that was similarly removed and reweighed. The increase in 
the amount of food consumed during the second exposure was taken as an index of decreased neophobia, an indicator that the mice formed a long-term memory of the novel taste.

To assess the development of a conditioned taste aversion to blueberry bar, we used a variation of the typical conditioned taste aversion protocol, adapted to solid food in nondeprived mice. Mice were randomly assigned to two groups $(n=5)$ and given $10 \mathrm{~min}$ access to blueberry bar followed by injection of either $\mathrm{LiCl}$ or $\mathrm{NaCl}(0.15 \mathrm{M}, 20 \mathrm{ml} / \mathrm{kg}$, i.p.). On the next day, mice were given a $10 \mathrm{~min}$ access to blueberry bar, and intakes were recorded, with decreased consumption relative to $\mathrm{NaCl}$ controls taken as an indicator of conditioned taste aversion.

Effects of MAP kinase blockade on acquisition of conditioned taste aversion. To test whether a conditioned taste aversion to a solid food is MAP kinase-dependent, as has been shown for saccharin (Berman et al., 1998), we used the selective MEK inhibitor SL327, a blood-brain barrierpermeant compound which, when given peripherally, has been shown to block other MAP kinase-dependent forms of learning such as fear conditioning (Atkins et al., 1998) and spatial learning (Selcher et al., 1999). Mice were randomly assigned to one of four groups: LiCl, DMSO$\mathrm{LiCl}$, SL327-LiCl, and $\mathrm{NaCl}(n=5$ per group). Two groups of mice were injected with either DMSO (0.1 ml, s.c.) or the MEK inhibitor SL327 (a kind gift of Dr. James Trzaskos; DuPont, Billerica, MA; $100 \mathrm{mg} / \mathrm{kg}$ in 0.1 $\mathrm{ml}$ DMSO, s.c.). One hour later DMSO-LiCl and SL327-LiCl mice, along with two uninjected groups $(\mathrm{LiCl}$ and $\mathrm{NaCl}$ ) were given $10 \mathrm{~min}$ access to blueberry bar. Conditioning intakes were measured and were not significantly different between groups, indicating that neither DMSO nor SL327, when given subcutaneously, suppresses food intake under these conditions (data not shown). LiCl, DMSO-LiCl, and SL327-LiCl mice were then injected with $\mathrm{LiCl}(0.15 \mathrm{M} ; 20 \mathrm{ml} / \mathrm{kg}$, i.p. $)$, and $\mathrm{NaCl}$ mice were injected with $\mathrm{NaCl}(0.15 \mathrm{M} ; 20 \mathrm{ml} / \mathrm{kg}$, i.p). The next day mice were given a $10 \mathrm{~min}$ test access to blueberry bar, and intakes were recorded. Increased intakes in the SL327 mice relative to the LiCl-injected mice were taken as an indicator of attenuation of conditioned taste aversion.

Slice preparation and ex vivo pharmacological experiments. We developed a method for preparing live slices through insular cortex using a variation of the typical method for preparing hippocampal slices for physiological recordings (Roberson et al., 1999). Male Swiss Webster mice weighing 30-35 gm were killed by cervical dislocation, and brains were removed rapidly and placed into ice-cold cutting solution containing (in mM): 110 sucrose, $60 \mathrm{NaCl}, 3 \mathrm{KCl}, 1.25 \mathrm{NaH}_{2} \mathrm{PO}_{4}, 28 \mathrm{NaHCO}_{3}$, $5 \mathrm{D}$-glucose, $0.5 \mathrm{CaCl}_{2}, 7 \mathrm{MgCl}_{2}$, and 0.6 ascorbate, saturated with $95 \%$ $\mathrm{O}_{2}$ and $5 \% \mathrm{CO}_{2}$. The rostral portion of the forebrain containing insular cortex was mounted on a vibratome stage, and $300 \mu \mathrm{m}$ slices were transferred to ice-cold cutting solution. Slices were taken through the insular cortex until the striatum appeared. After equilibration in a 1:1 mix of cutting solution and aCSF containing (in $\mathrm{mM}$ ): $125 \mathrm{NaCl}, 2.5 \mathrm{KCl}$, $1.25 \mathrm{NaH}_{2} \mathrm{PO}_{4}, 25 \mathrm{NaHCO}_{3}, 10$ D-glucose, $2 \mathrm{CaCl}_{2}$, and $1 \mathrm{MgCl}_{2}$, saturated with $95 \% \mathrm{O}_{2}$ and $5 \% \mathrm{CO}_{2}$ at room temperature for $20 \mathrm{~min}$, slices were then transferred to $\mathrm{aCSF}$ for another hour at room temperature. Slices were then transferred to a $32^{\circ} \mathrm{C}$ water bath where they were maintained for $1 \mathrm{hr}$. In slices treated with the selective MEK inhibitor U0126 (Promega, Madison, WI), the inhibitor was added at the beginning of this incubation. After $1 \mathrm{hr}$ of incubation, slices were treated with forskolin (50 $\mu \mathrm{M}$ with $100 \mu \mathrm{M}$ Ro20-1724, a cAMP phosphodiesterase inhibitor) for $1 \mathrm{hr}$. Slices were then removed, frozen on dry ice, and noncortical tissue was dissected away. In experiments to examine histone acetylation, the histone deacetylase inhibitor trichostatin A (Sigma, St. Louis, MO) was added to a final concentration of $500 \mathrm{ng} / \mathrm{ml}$ at the beginning of the $32^{\circ} \mathrm{C}$ incubation; samples were collected $2 \mathrm{hr}$ later. For all slices, sample preparation for Western blotting was identical to that described below.

Histone extraction and acid-urea gel electrophoresis. Samples were Dounce-homogenized as described below. The nuclear fraction was pelleted by centrifugation $\left(18,000 \times g ; 15 \mathrm{~min} ; 4^{\circ} \mathrm{C}\right)$ and the supernatant was saved for conventional SDS-PAGE. Histones were extracted from the nuclear pellet by the addition of five volumes $0.2 \mathrm{M} \mathrm{HCl}$ and $10 \%$ glycerol, and the insoluble fraction was pelleted by centrifugation $\left(18,000 \times g ; 30 \mathrm{~min} ; 4^{\circ} \mathrm{C}\right)$. Histones in the acid supernatant were precipitated with 10 volumes of ice-cold acetone followed by centrifugation $\left(18,000 \times g ; 30 \mathrm{~min} ; 4^{\circ} \mathrm{C}\right)$. The histone pellet was then resuspended in $9 \mathrm{M}$ urea, and aliquots were assayed for total protein by Coomassie assay. $\beta$-mercaptoethanol and acetic acid were added to a final concentration of $5 \%$. Samples were resolved on $15 \%$ acrylamide acid-urea gels (0.9 $\mathrm{M}$ acetic acid, $7 \mathrm{M}$ urea) and then transferred to polyvinylidene difluoride (PVDF) membrane and processed for acetylated lysine im- munoreactivity. Supernatant samples were processed as described below and assayed for acetylated lysine immunoreactivity by conventional SDSPAGE and Western blotting with an antibody to acetylated lysine.

Novel versus familiar taste time course. Mice were randomly assigned to one of two main groups: novel mice had no previous exposure to the tastant before testing; familiar mice had three previous exposures to the food on three successive days followed by $2 \mathrm{~d}$ without access to blueberry bar. At all times animals had ad libitum access to water and their usual chow. On test day, mice were given a $10 \mathrm{~min}$ access to a preweighed portion of blueberry bar that was then removed and weighed to record intakes. In the "novel" group, one group of mice was not given access to blueberry bar; these mice are the naive controls, and data obtained from them are plotted on the zero time point. In the "familiar" group, one group of mice did not receive a fourth access to blueberry bar; these mice represent the familiar control group and are plotted at the $96 \mathrm{hr}$ time point. After consumption and recording of intakes, mice were killed at the following time points: $0.5,1,2,6,12,24$, and $48 \mathrm{hr}$ after consumption ( $n=4$ per time point).

Sample preparation and Western blotting. Mice were killed by cervical dislocation, and brains were removed rapidly and frozen on dry ice. Brains were then dissected on a freezing stage at $-12^{\circ} \mathrm{C}$; briefly, a 14 ga punch was used to score a circular groove centered over the intersection of the middle cerebral artery and rhinal fissure. A 14 ga punch with a notched end was then used to lift out the cortical punch that fractured at the external capsule, leaving behind noncortical tissue. Punches were then homogenized in a hypotonic lysis buffer containing $10 \mathrm{~mm}$ Tris, $\mathrm{pH}$ 7.5, $1 \mathrm{~mm}$ EDTA, $2.5 \mathrm{~mm}$ sodium pyrophosphate, $1 \mathrm{~mm} \beta$-glycerophosphate, $1 \mathrm{~mm}$ sodium orthovanadate, $1 \mathrm{~mm}$ phenylmethylsulfonylfluoride, $1 \%$ protease inhibitor cocktail (Sigma), and 1\% Igepal CA-630. Protein concentration was determined by Coomassie assay (Pierce, Rockford, IL), and volumes were adjusted so that all samples were 2 $\mu \mathrm{g} / \mu \mathrm{l}$. SDS-PAGE sample buffer $(5 \times)$ was added to each sample and boiled, and $30 \mu \mathrm{g} /$ lane was loaded onto either 8 or $10 \%$ acrylamide gels, depending on the molecular weight of the protein being assayed. Samples were blotted onto PVDF membrane (Immobilon P; Millipore, Bedford, MA), blocked in 5\% nonfat dry milk in Tris-buffered saline containing $0.05 \%$ Tween 20 (TTBS) for $1 \mathrm{hr}$, and then probed with the following antibodies (all from Cell Signaling or the parent company, New England Biolabs, Beverly, MA): phospho-Raf (Ser259), phospho-MEK1/2 (Ser217/221), phospho-MAPK (Erk1/2) (Thr202/Tyr204), and phosphop90RSK (Ser381). To examine lysine acetyltransferase activity, blots were probed with an antibody to acetylated lysine; this antibody recognizes not only acetylated histones, but other HAT substrates such as p53 and PCAF when acetylated on $\epsilon$-amino groups of lysine residues. All antibodies were diluted 1:1000 in TTBS and 1\% milk and incubated for either $2 \mathrm{hr}$ at room temperature or overnight at $4^{\circ} \mathrm{C}$. Blots were then rinsed for $20 \mathrm{~min}$ in TTBS, incubated in HRP-conjugated anti-mouse or anti-rabbit (1:25,000 in 1\% milk and TTBS), rinsed, incubated in enhanced chemiluminescent substrate (SuperSignal Pico; Pierce), and exposed to film (Kodak BioMax; Eastman Kodak, Rochester, NY). Films were scanned, and densitometry performed as described previously (Roberson et al., 1999).

\section{RESULTS}

\section{Solid food in nondeprived animals elicits neophobia and supports a conditioned taste aversion}

Initial experiments were conducted using nondeprived mice that had ad libitum access to food and water. Mice were given access to a variety of solid foods, and we found that they consumed variable amounts of such foods as Cheetos (Frito-Lay, Dallas, TX), Froot Loops (Kellogg's, Battle Creek, MI), pizza, and ironically, cat food. Of the extensive number of foods tested, Nutri-Grain (Kellogg's) blueberry breakfast bars were chosen because mice appeared to prefer this over other foods tested (data not shown) and consumed this novel food even when nonfood-deprived. The availability of several other flavors also makes this food useful for future experiments designed to assess discrimination learning. Because these mice $(n=6)$ had been exposed to such a wide variety of novel tastes, they were later used to conduct an initial screening of insular cortex for changes in lysine acetylation (see below). 


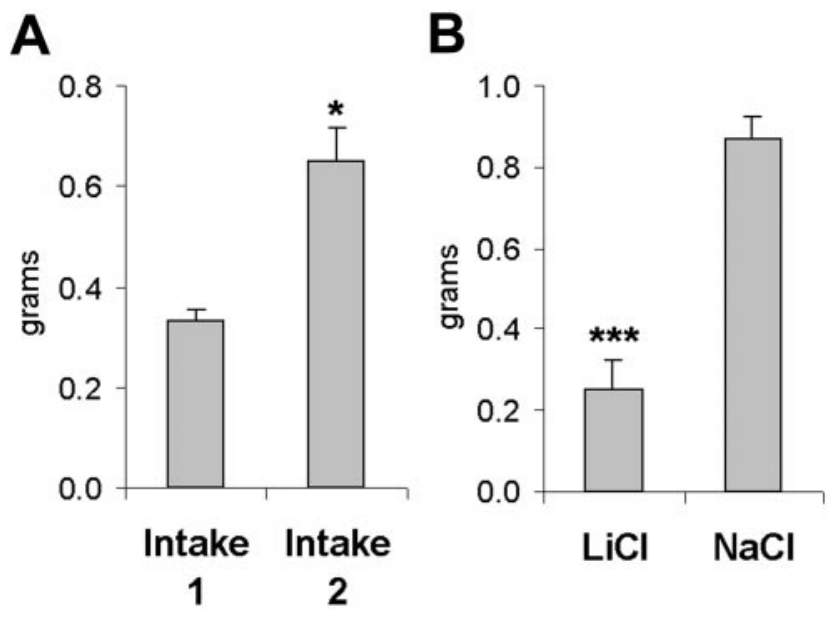

Figure 1. A, Neophobia during first access to a novel solid food is attenuated on second exposure. Mice were given $10 \mathrm{~min}$ access to NutriGrain blueberry bar, and intakes were recorded. Ten minute intakes on the second are significantly higher, demonstrating attenuation of neophobia through familiarization. ( ${ }^{*} p<0.05$ by one-way ANOVA). $B$, Pairing of solid novel food with $\mathrm{LiCl}$ produces a conditioned taste aversion. Mice injected with $\mathrm{LiCl}$ after a $10 \mathrm{~min}$ access to blueberry bar consume significantly less than $\mathrm{NaCl}$-injected when tested $(* * * p<0.001$ by oneway ANOVA).

Having demonstrated that mice would consume measurable quantities of a novel solid food, we then assessed neophobia, a characteristic reluctance to consume a novel foodstuff, to determine whether mice would form a lasting memory of the novel taste; if so, they would be expected to consume more on second exposure. We found that mice consumed a measurable quantity of blueberry bar during their initial $10 \mathrm{~min}$ access and that this first exposure was characterized by neophobia, as indicated by the observation that intakes were nearly double on the second access, a significant increase (Fig. $1 A$ ). These data indicate that the mice formed a memory of the novel taste after their first exposure, and this memory was expressed as an attenuation of neophobia on the second exposure, a result which is in line with other previously described taste learning paradigms (Bures, 1998).

Another attribute of taste learning is the ability to form selective robust aversions to a novel taste when it is paired with a malaise-inducing agent such as $\mathrm{LiCl}$. This is a highly adaptive form of learning that depends critically on the animal's ability to use the memory of the novel taste so that future encounters with a toxic and potentially lethal food can be avoided. As expected, test intakes of the novel taste that had been paired with $\mathrm{LiCl}$ were significantly suppressed relative to $\mathrm{NaCl}$ controls, demonstrating that the solid food stimulus supports a conditioned taste aversion (Fig. 1B). Along with the demonstration of attenuation of neophobia with familiarization, this experiment shows clearly that mice acquire a memory of the novel taste and suggests that the use of a novel solid food in nondeprived animals may be a useful tool to study mechanisms involved in novel taste memory.

\section{Conditioned taste aversion to a solid food is MAP kinase-dependent}

Because previous reports have shown that novel taste learning (Berman et al., 1998) and development of conditioned taste aversion to saccharin in water-deprived mice are MAP kinasedependent (Swank, 2000b), we wanted to confirm that this signal transduction cascade was also involved in taste learning using a novel solid food as the conditioned stimulus during conditioned

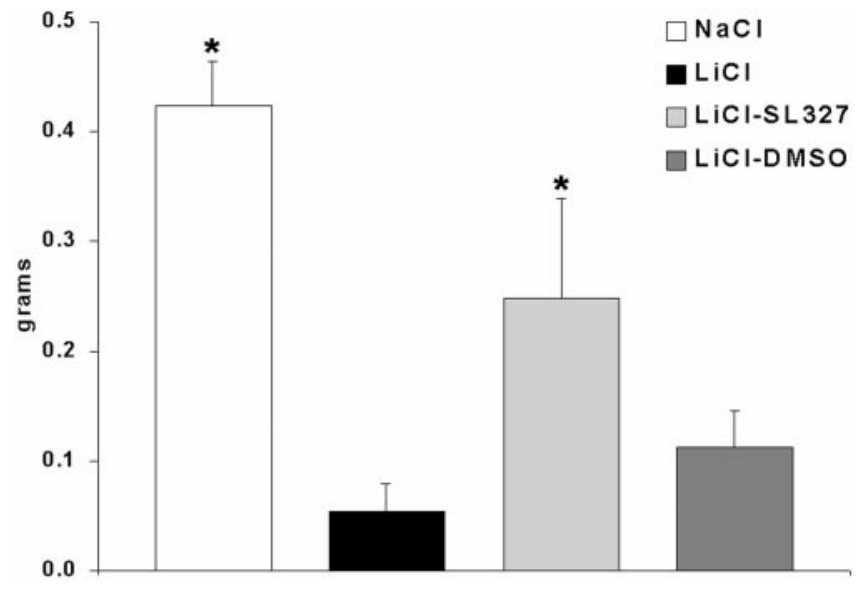

Figure 2. Memory for a novel food and acquisition of a conditioned taste aversion is MAPK-dependent. LiCl, LiCl-DMSO, and LiCl-SL327 mice all received 10 min access to blueberry bar followed by injection of $\mathrm{LiCl}$. $\mathrm{NaCl}$ mice received $\mathrm{NaCl}$ after blueberry bar. SL327 (100 mg/kg, s.c.) injected $1 \mathrm{hr}$ before blueberry bar significantly attenuates conditioned taste aversion, as shown by test intakes. $\left({ }^{*} p<0.05\right.$ relative to $\mathrm{LiCl}$ by ANOVA and Duncan interval).

taste aversion learning. Animals injected with $\mathrm{LiCl}$ developed a significant conditioned taste aversion to the blueberry bar, as shown by the markedly suppressed intakes relative to $\mathrm{NaCl}$ controls (Fig. 2). This was significantly attenuated by SL327 but not DMSO vehicle; only the SL327 and $\mathrm{NaCl}$ intakes were significantly greater than in $\mathrm{LiCl}$ mice. These data suggest, as has been documented previously for novel tastes in solution (Berman et al., 1998, 2000) that MAP kinase plays an important role in encoding a novel taste memory for a more complex solid food with additional odor and textural cues. The lack of complete blockade of conditioned taste aversion with MAP kinase inhibition is also consistent with previous reports using saccharin in solution (Berman et al., 1998).

\section{Regulation of histone acetylation in insular cortex}

In our first series of studies we demonstrated not only that mice form a memory for a novel solid food taste, but that this appears also to be MAP kinase-dependent. In this next phase we focused on histone acetyltransferase ${ }^{a}$ activity, an essential component of transcriptional regulation.

In our first experiment, we sought to confirm the presence of histone acetyltransferase activity in insular cortex. Although specific activators of histone acetyltransferases are not yet available, trichostatin A, a potent inhibitor of histone deacetylases (HDAC), has been shown to increase histone acetylation in a number of systems (Yoshida et al., 1990). We are unaware of any reports that have examined its effect in the brain, but we predicted that it was likely to act in a similar manner in the insular cortex. Not surprisingly, treatment of slices with trichostatin A produced a significant increase in acetylation of histones $\mathrm{H} 4$ and $\mathrm{H} 2 \mathrm{~A}$, but not H2B or H3 (Fig. 3). However, we also detected three prominent acetylated proteins when we used conventional SDS-PAGE

${ }^{a}$ The term histone acetyltransferase (HAT), although it would appear to refer specifically to the lysine acetylation of histones, is commonly used in the literature to refer also to lysine acetyltransferase activity responsible for acetylation of a rapidly growing list of other nonhistone substrates such as p53, CBP, and PCAF. We believe that the use of the term lysine (K) acetyltransferase (KAT) may be a more reasonable description of lysine acetyltransferases involved in acetylation of nonhistone substrates such as $\mathrm{p} 42 \mathrm{AcK}$ described in this report, and we reserve the use of the term histone acetyltransferase (HAT) for instances in which histones are demonstrated to be among the substrates that are acetylated. 


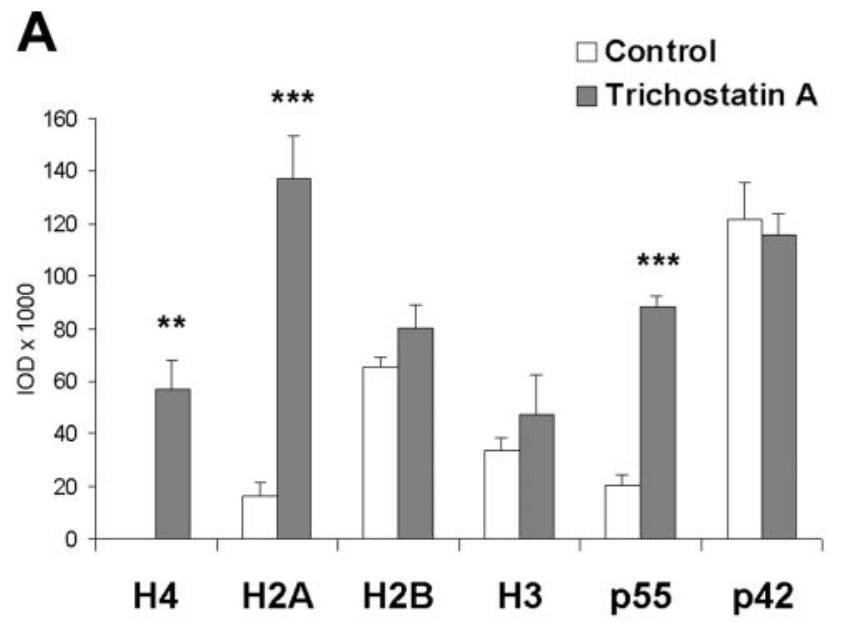

B

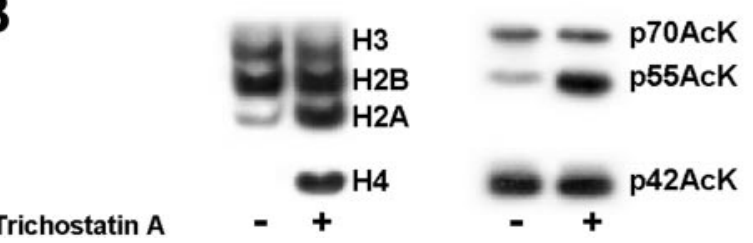

Figure 3. Effect of the histone deacetylase inhibitor trichostatin A on histone and non-nuclear protein acetylation in insular cortex slices. $A$, Two hour treatment with trichostatin A produced significant increases in acetylation of histones $\mathrm{H} 4, \mathrm{H} 2 \mathrm{~A}$, but not $\mathrm{H} 2 \mathrm{~B}$ or $\mathrm{H} 3$. Acetylation of an unknown protein, termed p55AcK, was also increased by trichostatin A. Acetylation of two other proteins, $\mathrm{p} 42 \mathrm{AcK}$ and $\mathrm{p} 70 \mathrm{AcK}$, was not altered by trichostatin $\mathrm{A}\left({ }^{*} p<0.05,{ }^{* *} p<0.01,{ }^{* * *} p<0.001\right.$ relative to controls by one-way ANOVA; $y$-axis: $I O D$, integrated optical density, i.e., pixel intensity $\times$ area). $B$, Representative blots showing acetylated lysine immunoreactivity after Western blots of histone extracts (left) or nonnuclear proteins (right).

to assay the supernatant from these samples; given their apparent molecular weights, these proteins are denoted p42AcK, p55AcK, and p70AcK. Lysine acetylation of p55AcK, but not p42AcK and p70AcK, was increased by trichostatin A. These data suggest that the acetylation state of p55AcK is regulated by HDAC, and by inference, histone acetyltransferases. These data suggest also that p42AcK and p70AcK are not regulated by histone acetyltransferases; however, a differential sensitivity to HDAC could account for the lack of altered acetylation in these two proteins. Taken together, our findings in this first experiment demonstrate the existence of both histone acetyltransferase and lysine acetyltransferase activity in insular cortex, as evidenced by lysine acetylation of several proteins and a regulation of histone acetylation by inhibition of histone deacetylases.

\section{Positive and negative coupling of two distinct lysine acetylation pathways to the MAP kinase cascade}

To continue our investigations we evaluated possible upstream regulators of lysine acetyltransferase and histone acetyltransferase activity in insular cortex slices. Given the differences in sensitivity to HDAC inhibitors, we were particularly interested in possible differential regulation of p42AcK, p55AcK, and p70AcK. Because ERK/MAP kinase has been implicated in regulating histone acetyltransferase activity in other systems (Ait et al., 1999; Kawahara et al., 1999), we chose to test this pathway first. As a first experiment, we needed to examine ERK/MAPK regulation in insular cortex, and we hypothesized that PKA might regulate MAPK in insular cortex as it does in hippocampus
(Roberson et al., 1999). Presumed activation of PKA by forskolin produced a robust activation of the MAP kinase cascade, as shown by highly significant increases in phosphorylation of both the 42 and $44 \mathrm{kDa}$ isoforms of MAPK (Fig. $4 A$ ). Treatment of slices with the selective MEK inhibitor U0126 not only abolished the forskolin response, but also reduced basal phospho-MAPK to levels undetectable by Western blot (Fig. 4D). These data indicate that the cAMP cascade regulates MAPK in insular cortex.

Our next set of experiments indicated that MAPK can regulate lysine acetyltransferase activity in insular cortex. Acetylation of p42AcK was significantly increased by forskolin-treated insular cortex slices, and this was blocked by the specific MEK inhibitor U0126 (Fig. 4B), demonstrating that acetylation of p42AcK is MAP kinase-dependent. In contrast, acetylation of p55AcK was significantly increased by the MEK inhibitor U0126 independent of forskolin, suggesting that the MAP kinase cascade exerts an inhibitory control over the histone acetyltransferases responsible for acetylation of p55AcK. p70AcK was not affected by any treatment (data not shown). These data, like those obtained after treatment with trichostatin A, suggest a differential regulation of p42- and p55AcK. Importantly, they indicate a positive regulation of a novel lysine acetyltransferase activity by the ERK/MAPK cascade in insular cortex and a possible negative regulation of histone acetyltransferase activity by the ERK/MAPK cascade in the insular cortex.

\section{Previous exposure to novel tastes increases lysine acetylation of p42AcK and p55AcK in insular cortex}

We next sought to determine whether novel taste learning is associated with activation of histone acetyltransferase and lysine acetyltransferase in insular cortex. An initial pilot experiment examined insular cortices of mice that had been exposed to a variety of novel tastes (see Results), to determine whether novel taste learning is associated with lysine acetyltransferase activation. Western blots of acutely prepared insular cortex revealed the same three prominent acetylated proteins of $\sim 42,55$, and $70 \mathrm{kDa}$ that were observed in insular cortex homogenates obtained from in vitro slice experiments (Fig. $5 A$ ). Compared with naive controls, mice that had been used to screen for novel solid food preferences showed significant increases in lysine acetylation of p42AcK and p55AcK; p70AcK did not appear to be altered by taste experience (Fig. $5 B$ ). These pilot data compelled us to perform the following controlled experiment to compare novel with familiarized taste at a number of time points after taste exposure.

\section{A single exposure to a novel taste produces increased lysine acetylation of p42- and p55AcK}

A single $10 \mathrm{~min}$ exposure to novel taste produced significant increases in lysine acetylation of both p42AcK and p55AcK. (Fig. $6 A, B)$. Representative blots are shown in Figure $6 C$. Given that the slice experiments revealed two distinct lysine acetyltransferase pathways responsible for acetylating $\mathrm{p} 42 \mathrm{AcK}$ and $\mathrm{p} 55 \mathrm{AcK}$, perhaps not surprisingly the kinetics of acetylation of these two proteins were found to be different. p42AcK showed an unusual biphasic response: acetylation was significantly increased relative to naive controls at 1 and $2 \mathrm{hr}$, then decreased to control levels followed by significant increases at 12, 24, and $48 \mathrm{hr}$ (Fig. 6A). In contrast, p55AcK showed a more prolonged response, with acetylation being significantly increased at all time points out to $12 \mathrm{hr}$, followed by a return to baseline at 24 and $48 \mathrm{hr}$ (Fig. 6B).

We next sought to determine what effect repeated presenta- 
A
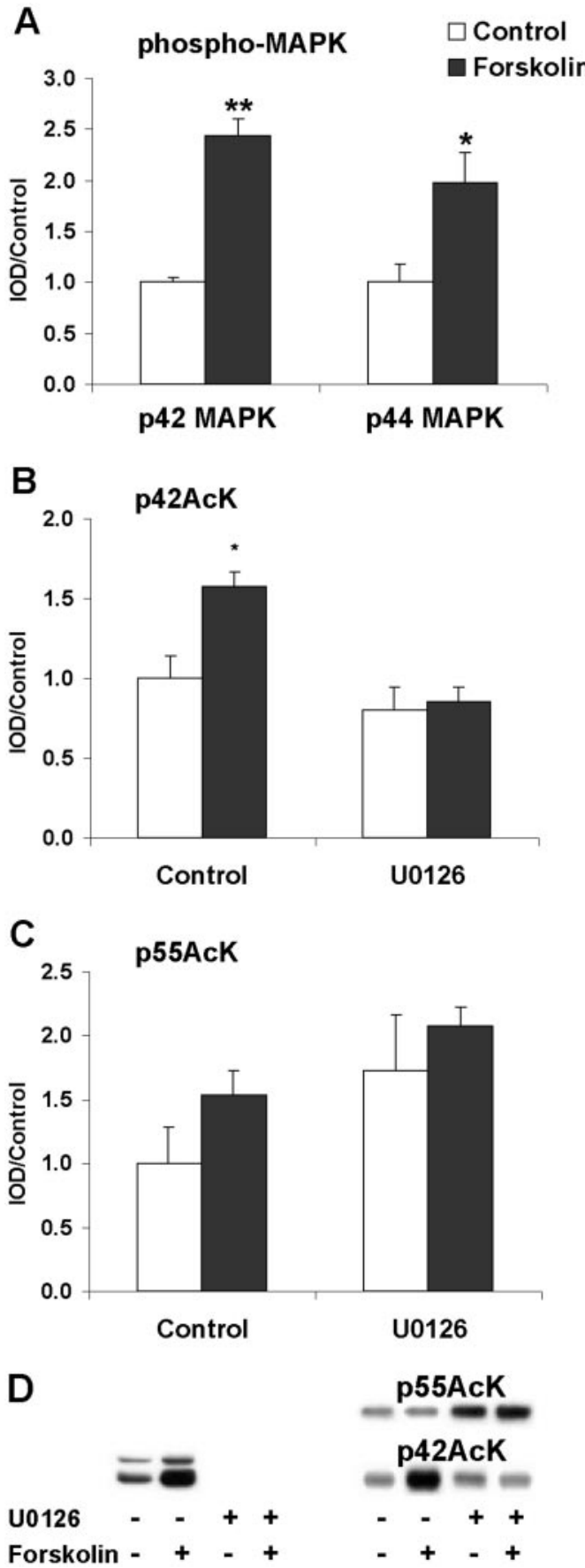

Figure 4. Effects of forskolin activation of PKA on MAPK activation and lysine acetylation. $A$, Forskolin produces a significant activation of both p42 and p44 isoforms of MAP kinase. The selective MEK inhibitor U0126 completely abolishes phospho-MAPK immunoreactivity $(D)$ and is not plotted here. Two-way ANOVA revealed a significant main effect of forskolin on phosphorylation of both p42ERK $(p<0.001)$ and p44ERK $(p<0.05)$; there was also a significant main effect of U0126 $(p<0.001)$. $B$, Activation of the MAPK cascade elicits an increase in lysine acetylation of p42AcK that is blocked by U0126. Two-way ANOVA revealed a significant main effect of both forskolin $(p<0.05)$ and U0126 $(p<0.01)$. Post hoc Dunnett analysis revealed that forskolin control acetylation was significantly greater than forskolin U0126 $(p<0.05)$. $C$, Inhibition of the MAPK cascade by U0126 causes increased acetylation of p55AcK. Twoway ANOVA revealed a significant main effect of U0126 $(p<0.05)$ but not forskolin. $D$, Representative Western blots of phospho-MAPK (left) and acetylated lysine (right). Note the complete absence of phosphoMAPK immunoreactivity in U0126 slices.
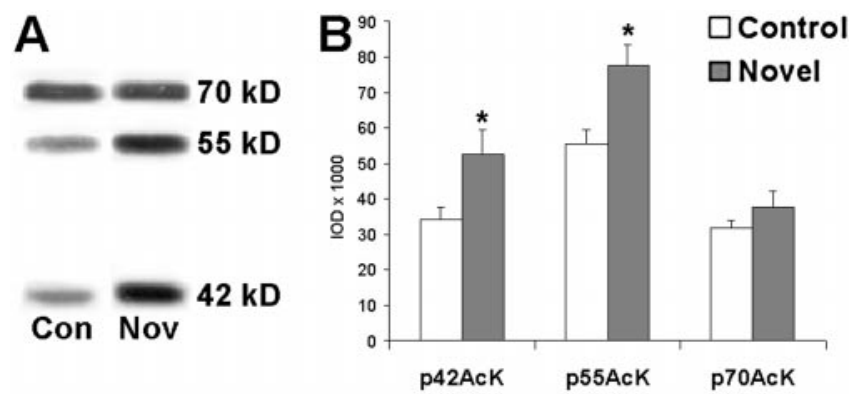

Figure 5. Acetylation of a 42 and $55 \mathrm{kDa}$ protein, but not a $70 \mathrm{kDa}$ protein, is altered by previous novel taste exposure. Mice that had been used to screen for solid food preferences were used for an initial screen for lysine acetylation in insular cortex. $A$, Western blot revealed three prominent acetylated proteins in both novel and naive mice. $B$, Lysine acetylation of both the 42 and $55 \mathrm{kDa}$ proteins was shown to be increased after several previous exposures to novel foods $(p<0.05$ by one-way ANOVA).

tions of the taste stimulus would have on insular cortex lysine acetylation. Similar to the response of p42 and p55AcK acetylation after a single presentation of novel taste, repeated taste presentation (familiar) elicited significantly elevated lysine acetylation of both $\mathrm{p} 42 \mathrm{AcK}$ and $\mathrm{p} 55 \mathrm{AcK}$ relative to naive controls (Fig. 6A,B). These data are consistent with our observation that novel taste elicits lysine acetylation in insular cortex, in that repeated presentation of the same stimulus also elicits altered lysine acetylation. Although interpretation of our findings is speculative at present, these data may also suggest that lysine acetylation is chronically increased when a formerly novel taste has become familiar, i.e., when long-term memory for that taste has been acquired. In this scenario, the acute response to novel taste is potentially associated with acquisition and consolidation. However, additional behavioral experiments will be needed to address these issues.

\section{Activation of the MAPK/ERK pathway by novel taste in insular cortex}

Given that the in vitro studies described above demonstrated that acetylation of $\mathrm{p} 42 \mathrm{AcK}$ is regulated by MAP kinase, we hypothesized that increased lysine acetylation of $\mathrm{p} 42 \mathrm{AcK}$ was a result of MAP kinase activation in the insular cortex. This hypothesis was especially appealing, because MAP kinase activation in the insular cortex has already been shown after novel taste learning (Berman et al., 1998; Swank, 2000a). Similar to results previously reported using novel saccharin in water-deprived rats, the ERK/ MAP kinase pathway was activated in insular cortex after the novel solid food taste stimulus. Peak phosphorylation of $\mathrm{p} 42$ (Fig. $7 A$ ) and p44 MAPK (Fig. 7B) occurred within 2 and $6 \mathrm{hr}$, respectively, after novel taste. Phosphorylation then dropped toward baseline at $12 \mathrm{hr}$, followed by significant increases at 24 and $48 \mathrm{hr}$. These findings demonstrate MAP kinase activation in insular cortex in response to novel solid food taste presentation.

Similar to the response to the novel taste, there was a significant increase, relative to naive controls, in phosphorylation of both p42 and p44 MAPK at all time points after repeated exposure to the taste, i.e., taste familiarization. However, there was no significant additional increase in MAPK phosphorylation with a fourth exposure. These data suggest that once the mice have retained a long-term memory of the novel taste, the now-familiar taste elicits minimal additional changes in MAPK activation. However, overall levels of MAP kinase phosphorylation in familiarized animals 

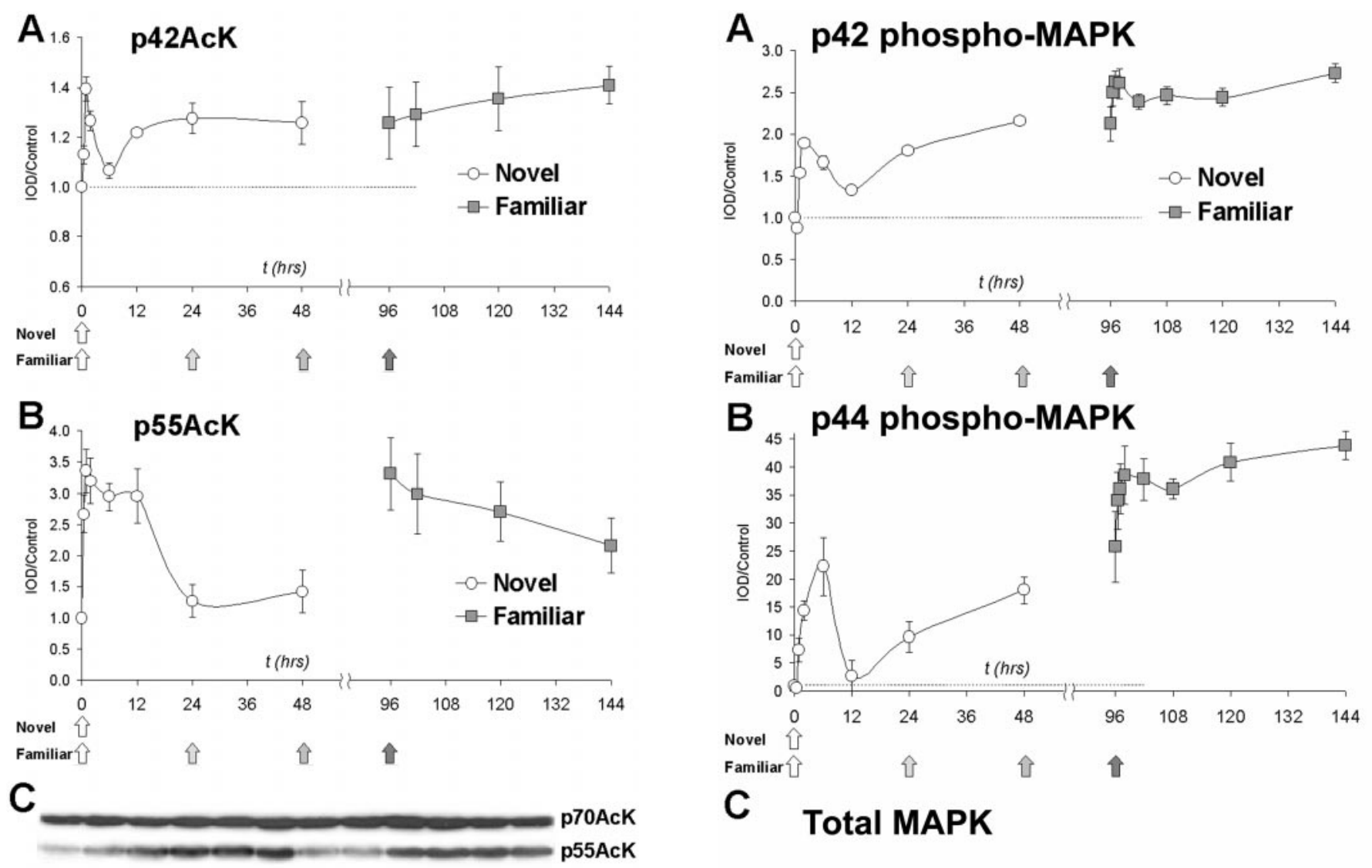

C Total MAPK

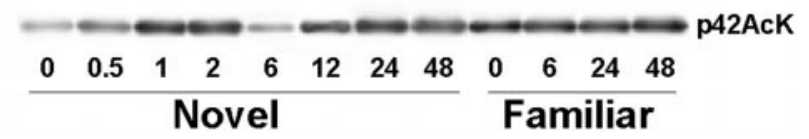

Figure 6. Acetylation of p42AcK $(A)$ and p55AcK $(B)$ after novel and familiar taste. Novel mice were given a single $10 \mathrm{~min}$ access to blueberry bar (denoted by arrow at $t=0 \mathrm{hr}$ ), and time of killing is indicated by the $x$-axis. The data point at $t=0 \mathrm{hr}$ represents the control group of naive mice that did not receive novel food. Familiar mice received three previous exposures at 0,24 , and $48 \mathrm{hr}$ (denoted by arrows) followed by an additional $48 \mathrm{hr}$ before the fourth exposure at $t=96 \mathrm{hr}$. The data point at $t=96 \mathrm{hr}$ represents mice with three previous exposures only and is the control group for the familiar groups. All data are expressed as integrated optical density (area $\times$ pixel intensity) normalized to the $t=0$ control mice. $A$, Lysine acetylation of $\mathrm{p} 42 \mathrm{AcK}$ shows a biphasic response in the novel group and a persistent increase in the familiar group. Two-way ANOVA showed significant main effects of both group (novel vs familiar; $p<0.01)$ and time $(p<0.05)$. Post hoc Dunnett analysis revealed a significant increase in p42AcK acetylation in the novel group at $1,2,12$, and $24 \mathrm{hr}$. Relative to $t=96 \mathrm{hr}$ controls, there was no significant change in the familiar group. B, Lysine acetylation of p55AcK. Two-way ANOVA revealed significant effects of both group (novel vs familiar; $p<0.001$ ) and time $(p<0.001)$. Post hoc Dunnett analysis revealed significant increases in acetylation of p55AcK at all time points up to $12 \mathrm{hr}$, but not at 24 and $48 \mathrm{hr}$. There was no significant change in acetylation of the familiar group at any time points relative to $t=96 \mathrm{hr}$. $C$, Representative immunoblots showing changes in acetylation of $\mathrm{p} 42 \mathrm{AcK}$ and $\mathrm{p} 55 \mathrm{AcK}$. Note that there is no change in the acetylation of p70AcK.

were significantly increased relative to naive controls, suggesting that persistent activation of MAPK is associated with retention.

The kinetics of MAPK phosphorylation after both single and repeated exposures to a novel taste are similar to the acetylation of $\mathrm{p} 42 \mathrm{AcK}$. In both cases, a single novel taste produces a biphasic activation; the effect of repeated presentation leading to familiar-

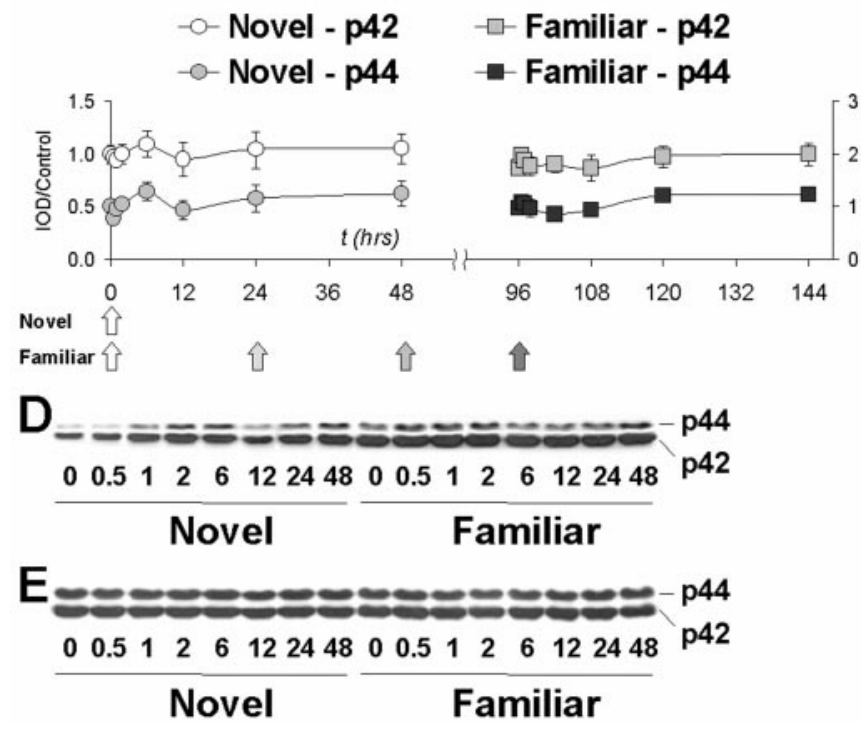

Figure 7. Phosphorylation of $\mathrm{p} 42$ and $\mathrm{p} 44$ MAP kinase after novel or familiar taste. $A, B$, Two-way ANOVA revealed significant main effects of both group (novel vs familiar, $p<0.001)$ and time $(p<0.001)$ for both MAPK isoforms. A, Post hoc Dunnett analysis of p42ERK revealed significant increases in phosphorylation at $1,2,6,24$, and $48 \mathrm{hr} . B$, p44 MAPK phosphorylation was significantly increased at 2,6 , and $48 \mathrm{hr}$. $A$, $B$, For both MAPK isoforms, phosphorylation was significantly increased at the $144 \mathrm{hr}$ time point; this is $48 \mathrm{hr}$ after the fourth and final exposure to the now-familiar taste. $C$, Total MAPK levels are unchanged in both the novel and familiar groups for both MAPK isoforms. Normalized p42 values are plotted against the left $y$-axis, and p44 values against the right $y$-axis. Two-way ANOVA revealed that there was no significant effect of either group (novel vs familiar) or time. $D, E$, Representative immunoblots showing phospho-MAPK and total MAPK, respectively. 
ization is to elicit increased activity that it not significantly altered by additional exposure to a familiar taste. Taken together with the in vitro insular cortex slice experiments, these data are consistent with the hypothesis that acetylation in vivo of p42AcK may be regulated by MAP kinase. Additional future experiments in vivo will be necessary to test this hypothesis directly.

Equally important, our data indicate an interesting long-term regulation of ERK/MAPK in insular cortex after novel taste exposure. In the next series of experiments, we sought to gain some insight into the basis for this long-lasting activation of ERK/MAPK.

\section{Changes in phospho-MAPK are not attributable to an increase in total MAPK}

Although phospho-MAPK levels changed dramatically across the different groups, total MAPK levels were not significantly altered by either single or repeated presentations of novel food at any time point (Fig. $7 C$ ). These data demonstrate that the increases in MAPK phosphorylation seen here are not attributable to changes in the total amount of MAPK.

\section{Activation of the entire ERK/MAPK cascade by novel taste}

Regulation of ERK/MAPK activity is complex, and there are several upstream activators. We sought to determine whether the two upstream regulators of MAPK in the canonical MAPK cascade, MEK and Raf, were also activated in insular cortex during novel taste learning. Both are positively regulated by phosphorylation, and inferred activity can, like MAPK, be measured by Western blots with phosphospecific antibodies.

Phosphorylation of MEK showed similar kinetics to that of MAP kinase. In the novel group, phospho-MEK was significantly increased at 1 and $2 \mathrm{hr}$, declined, and was then significantly increased at 24 and $48 \mathrm{hr}$. Repeated taste presentation also produced overall significant increases in phospho-MEK relative to the naive controls, but again, as with MAPK, additional presentations did not elicit any further increases in MEK phosphorylation relative to the familiar control $(t=96 \mathrm{hr}$ ) (Fig. 8A).

Raf is an upstream activator of MEK that is also positively regulated by phosphorylation. The kinetics of Raf phosphorylation were similar to that of MAP kinase during the first wave of activation (Fig. $8 B$ ); novel taste produced significant elevations of phospho-Raf at $0.5,1,2,6$, and $12 \mathrm{hr}$. However, one difference here is the lack of a second wave of activation of Raf; phosphorylation of Raf after novel taste declined at $12 \mathrm{hr}$ and was not significantly different from naive controls at 24 and $48 \mathrm{hr}$. Similarly, although levels of phospho-Raf were significantly increased by repeated taste presentation, there was a significant decline 48 hr after the fourth taste presentation (144 hr time point). These data suggest the interesting possibility that the prolonged phase of MEK/MAPK activation we observed may be attributable to a Raf-independent mechanism.

In addition to upstream activators of ERK/MAPK, we also sought to investigate the possible regulation of MAPK substrates. We focused on p90RSK because it is a MAPK substrate whose activity is regulated by phosphorylation and because p90RSK can couple MAPK to phosphorylation of such downstream effectors as CREB. The kinetics of p90RSK phosphorylation were similar to those described above, although the magnitude of the response here was much greater than for other components of the MAP kinase cascade (Fig. 8C). Representative blots for phospho-Raf, phospho-MEK, and phospho-p90RSK are shown in Figure $8 D$.

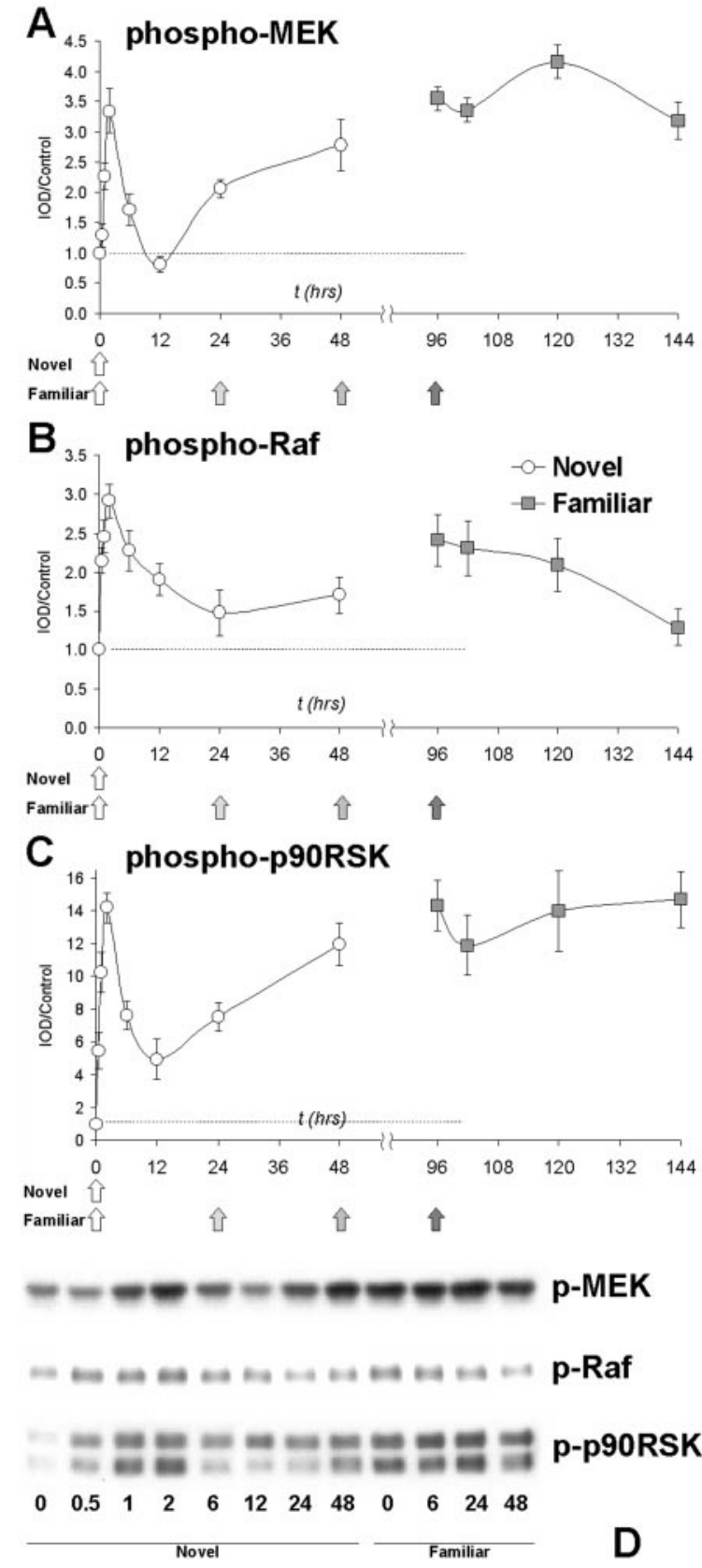

Figure 8. Phosphorylation of MAP kinase cascade elements MEK, Raf, and p90 RSK. $A$, phospho-MEK shows a biphasic response. Two-way ANOVA revealed a significant main effect of both group (novel vs familiar; $p<0.001)$ and time $(p<0.001)$. Post hoc Dunnett analysis revealed significant increases in phospho-MEK at 1, 2, 24, and $48 \mathrm{hr}$ in the novel group but no significant effect of time in the familiar group. $B$, Phosphorylation of Raf does not show the biphasic response seen with p42AcK and phospho-MAPK. Two-way ANOVA revealed a significant main effect of both group (novel vs familiar; $p<0.05)$ and time $(p<$ 0.001 ). phospho-Raf was significantly increased at $0.5,1,2,6$, and $12 \mathrm{hr}$, but then declined to baseline at 24 and $48 \mathrm{hr}$. Within the familiar group, phospho-Raf levels at $t=144 \mathrm{hr}$ ( $48 \mathrm{hr}$ after the fourth taste exposure) were significantly depressed relative to the $t=96 \mathrm{hr}$ control. $C$, phosphop90RSK also shows a biphasic response to novel taste. Two-way ANOVA revealed significant main effects of both group (novel vs familiar; $p<$ $0.001)$ and time $(p<0.001)$. Within the novel group, phosphorylation was significantly increased at all time points, although the plot shows the characteristic biphasic response. As with other members of the MAPK cascade, there was no significant effect of time within the familiar group. $D$, Representative immunoblots showing phospho-MEK, phospho-Raf, and phospho-p90RSK immunoreactivity. 
Novel taste produced significant increases in phosphorylation of p90RSK at all time points; however, the response shows a similar biphasic response, declining at $12 \mathrm{hr}$, and then increasing at 24 and $48 \mathrm{hr}$. Repeated taste presentations produced significant increases in phospho-p90RSK, but there was no significant additional increase with a fourth exposure relative to the familiar controls $(t=96 \mathrm{hr})$. The finding that p90RSK phosphorylation followed similar kinetics as MAPK is interesting for several reasons. As a substrate of MAPK, it allows one to track MAPK activity and confirms the MAPK activation observed in our earlier experiments. Furthermore, as a kinase that regulates phosphorylation of the transcription factor CREB, it provides a possible immediate link to altered gene expression mediated by MAP kinase.

\section{DISCUSSION}

\section{Novel taste memory is associated with long-lasting changes in lysine acetylation of p42AcK and p55AcK}

A single $10 \mathrm{~min}$ exposure to a novel taste is sufficient to produce a memory for that taste, as shown by the attenuation of neophobia and the capacity for acquisition of a conditioned taste aversion to that taste. Insular cortex histone and lysine acetyltransferase activities were both regulated by novel taste but showed distinctly different kinetics. Lysine acetylation of p55AcK showed an immediate and prolonged response up to $12 \mathrm{hr}$, and then levels declined to baseline. Lysine acetylation of $\mathrm{p} 42 \mathrm{AcK}$, on the other hand, showed an unusual biphasic response which has not, to our knowledge, been reported for any biochemical marker in any learning or memory paradigm to date. Although acetylation decreased at $6 \mathrm{hr}$, levels were significantly elevated again even at 48 hr. The suggestion from these data that such a late response may be involved in long-term memory formation is supported by the findings that acetylation is persistently elevated in the insular cortex of animals receiving multiple presentations of the novel taste. The possibility that the long-lasting activation of p42AcK and p55AcK after repeated presentations is a result of familiarization, i.e., consolidation of the memory of the novel taste, is a tantalizing hypothesis. One important consideration to note is that the immunoreactive 42 and $55 \mathrm{kDa}$ bands seen here may actually represent multiple acetylation substrates that comigrate on SDS-PAGE, and the kinetics seen here may actually represent different substrates that are acetylated at different time points.

\section{Novel taste memory is associated with biphasic activation of the entire ERK/MAPK cascade, and familiarization produces a persistent activation of the ERK/MAPK cascade}

Although the biphasic response of p42AcK is unusual, demonstration of coupling to the MAP kinase cascade is suggested by the finding of a similar activation of the entire ERK/MAPK cascade. Novel taste produced a biphasic increase in MAP kinase phosphorylation, and repeated taste presentation produced a persistently elevated phosphorylation. Both the upstream activator MEK and the downstream substrate p90RSK showed similar kinetics, demonstrating activation of the entire ERK/MAPK cascade.

The coordinate regulation of MEK suggests that future experiments be performed to examine the functional role of this later activation, along with the possible in vivo MAPK-dependent acetylation of p42Ack. These experiments might conceivably use the peripherally administered MEK inhibitor SL327, which has been shown here to block conditioned taste aversion when given shortly before the novel taste. Given the complex kinetics of
MAPK, lysine acetyltransferase (KAT), and HAT activation, along with the short half-life of such MEK inhibitors as SL327, such experiments will require that the dosing regimen is optimized to block various stages of ERK activation, including those that persist for hours or days after novel taste presentation. At this time, both the behavioral necessity of the later activation of the MAPK cascade, along with its role in lysine acetylation in insular cortex, remain to be tested.

A dichotomy in the biphasic response of ERK/MAPK cascade components is seen when comparing phosphorylation of Raf, an immediate upstream activator of MEK, with the phosphorylation of MEK itself. Phosphorylation of Raf appears to show only the first wave of activation and is not significantly elevated in the novel group after $12 \mathrm{hr}$. This may provide a clue to the biphasic activation of MEK/MAPK, because a different MEK activator, perhaps Rap, may be recruited for later MAPK activation. It is also plausible that this late phase of activation may depend on transcriptional activation by the first wave of MAPK activation. Although this has not been reported in the brain, such a regulatory system has been seen with MAP kinase activation in oocytes, which exhibit a biphasic activation of MAP kinase; activation of MAP kinase stimulates transcriptional activation of the kinase c-Mos, which can activate MAP kinase via phosphorylation of MEK, resulting in a positive feedback loop between c-Mos and MAP kinase (Fisher et al., 2000). It will be interesting to determine whether such a self-reinforcing feedback loop, a mnemogenic chemical reaction by the criteria of Roberson and Sweatt (1999), exists in the insular cortex after novel taste learning.

This unusual biphasic response has not been previously reported for such MAP kinase-dependent learning and memory paradigms as novel taste learning (Berman et al., 1998) spatial learning (Blum et al., 1999; Selcher et al., 1999), or fear conditioning (Atkins et al., 1998; Schafe et al., 2000). However, in none of these previous reports was MAP kinase examined for longer than 2 or $3 \mathrm{hr}$ after behavioral manipulations leading to MAPK activation, thus raising the possibility that the biphasic activation seen here is not unique to our specific paradigm. Further examination of the long-term kinetics of MAP kinase activation in other learning and memory paradigms will be necessary to address this intriguing possibility.

\section{Histone acetyltransferase and lysine acetyltransferase are positively and negatively regulated by MAP kinase}

Histone and lysine acetyltransferase activity leading to lysine acetylation of p42AcK and p55AcK appear to be regulated by separate and distinct pathways. Lysine acetylation of p42AcK was found to be MAPK-dependent in our in vitro insular cortex slice experiments. The possibility of ERK regulation of $\mathrm{p} 42 \mathrm{AcK}$ is further supported by the similar kinetics of p42AcK and MAPK in vivo by both novel and familiar taste. Lysine acetylation of p42AcK does not appear to be regulated by histone acetyltransferases, as shown by the lack of effect of trichostatin A on p42AcK acetylation. This finding raises the interesting possibility that in addition to histone acetyltransferases, a novel lysine acetyltransferase activity may be active in the brain, and whose function is to regulate, by lysine acetylation, nonhistone substrates. We can only speculate at this point how lysine acetylation might regulate nonhistone substrates, but neutralization of positively charged lysine residues might be considered analogous to the dramatic changes that occur with phosphorylation: by altering the charge, conformational changes and alterations of protein-protein interactions could have profound effects on the function of lysine 
acetyltransferase substrates. We are presently working to purify and sequence both the $\mathrm{p} 42 \mathrm{AcK}$ and $\mathrm{p} 55 \mathrm{AcK}$ proteins to elucidate their potential roles in neuronal plasticity underlying novel taste learning.

In contrast to $\mathrm{p} 42 \mathrm{AcK}$, acetylation of $\mathrm{p} 55 \mathrm{AcK}$ is regulated by histone acetyltransferases, because trichostatin A caused a coordinate increase in acetylation of histones and p55AcK. It should be noted here that although histone acetylation was measured in slices, because of the amount of tissue required to perform the histone extraction, we were unable to measure this in insular cortex punches obtained in vivo. However, given that in slices p55AcK acetylation occurred in parallel with that of histones, this unknown substrate may provide a useful assay for histone acetyltransferase activity in vivo. Slice experiments also demonstrated that inhibition of the MAPK cascade with the selective MEK inhibitor U0126 elicited significant increases in p55AcK acetylation, suggesting that the MAPK cascade exerts an inhibitory control over histone acetyltransferase activity in the insular cortex. However, we remain cautious in our interpretation of these findings, because this increase in $\mathrm{p} 55 \mathrm{AcK}$ acetylation occurs after total obliteration of basal phospho-MAPK, an event that is unlikely to occur in vivo.

\section{Solid food in nondeprived mice is a useful tool for studying novel taste memory}

Although not used to any great extent before this report, we note the utility of a solid food stimulus in the study of molecular and biochemical mechanisms of learning and memory. This paradigm has several features that make it desirable for taste learning studies. First, its simplicity allows for high throughput, because animals do not have to be maintained on any food or water deprivation schedule. Second, given that water deprivation produces anorexia and results in large changes in gene expression in feeding-related areas of the brain (Watts, 2000), the use of nonwater-deprived animals may allow biochemical measurements that may be more representative of the animal in its natural state. Furthermore, a novel solid food stimulus may represent a reasonable reiteration of an ethologically relevant stimulus that a mouse, a foraging animal, may encounter. Therefore, a novel solid food, by mimicking what the mouse evolved to deal with in its natural environment, may elicit particularly robust biochemical changes in the insular cortex.

\section{Long-lasting changes in biochemical activity during novel taste learning may represent changes in gene expression}

Perhaps the most striking finding of this paper is that a single 10 min exposure to a novel taste stimulus produces dramatic biochemical changes that persist for many hours after the initial stimulus. Activation of the entire MAP kinase pathway, lysine acetylation, and to a lesser extent, histone acetyltransferase activity, all show dramatic changes as a result of a novel taste stimulus. Furthermore, three previous exposures to a novel taste are sufficient to produce persistent activation of all three pathways for at least $48 \mathrm{hr}$ after the last exposure. Although many different scenarios may be posited, we believe that transcriptional activation and changes in gene expression are likely to subserve some of these persistent biochemical changes. Although not examined here, one likely mediator is CREB, a transcription factor implicated in a number of learning and memory paradigms, and a substrate of the MAPK- activated kinase p90RSK. Hopefully future experiments will allow the testing of this interesting hypothesis.

\section{REFERENCES}

Ait SA, Carlisi D, Ramirez S, Upegui-Gonzalez LC, Duquet A, Robin P, Rudkin B, Harel-Bellan A, Trouche D (1999) Phosphorylation by p44 MAP Kinase/ERK1 stimulates CBP histone acetyltransferase activity in vitro. Biochem Biophys Res Commun 262:157-162.

Alberini CM (1999) Genes to remember. J Exp Biol 202:2887-2891.

Allfrey VG, Faulkner R, Mirsky AE (1964) Acetylation and methylation of histones and their possible role in the regulation of RNA synthesis. Proc Natl Acad Sci USA 61:786-794.

Atkins CM, Selcher JC, Petraitis JJ, Trzaskos JM, Sweatt JD (1998) The MAPK cascade is required for mammalian associative learning. Nat Neurosci 1:602-609.

Bailey CH, Bartsch D, Kandel ER (1996) Toward a molecular definition of long-term memory storage. Proc Natl Acad Sci USA 93:13445-13452.

Berman DE, Hazvi S, Rosenblum K, Seger R, Dudai Y (1998) Specific and differential activation of mitogen-activated protein kinase cascades by unfamiliar taste in the insular cortex of the behaving rat. J Neurosci 18:10037-10044.

Berman DE, Hazvi S, Neduva V, Dudai Y (2000) The role of identified neurotransmitter systems in the response of insular cortex to unfamiliar taste: activation of ERK1-2 and formation of a memory trace. J Neurosci 20:7017-7023.

Blum S, Moore AN, Adams F, Dash PK (1999) A mitogen-activated protein kinase cascade in the CA1/CA2 subfield of the dorsal hippocampus is essential for long-term spatial memory. J Neurosci 19:3535-3544.

Bures JB-RFYT (1998) Conditioned taste aversion. Memory of a special kind. Oxford: Oxford UP.

Davie JR, Spencer VA (1999) Control of histone modifications. J Cell Biochem [Suppl] 32-33:141-148.

Fisher DL, Mandart E, Doree M (2000) Hsp90 is required for c-Mos activation and biphasic MAP kinase activation in Xenopus oocytes. EMBO J 19:1516-1524.

Impey S, Smith DM, Obrietan K, Donahue R, Wade C, Storm DR (1998) Stimulation of cAMP response element (CRE)-mediated transcription during contextual learning. Nat Neurosci 1:595-601.

Kawahara K, Watanabe S, Ohshima T, Soejima Y, Oishi T, Aratani S, Nakata M, Shibata M, Inoue K, Amano T, Fujii R, Yanai K, Hagiwara M, Fukamizu A, Maruyama I, Nakajima T (1999) Hypernuclear acetylation in atherosclerotic lesions and activated vascular smooth muscle cells. Biochem Biophys Res Commun 266:417-424.

Kouzarides T (2000) Acetylation: a regulatory modification to rival phosphorylation? EMBO J 19:1176-1179.

Martinez-Balbas MA, Bannister AJ, Martin K, Haus-Seuffert P, Meisterernst M, Kouzarides T (1998) The acetyltransferase activity of CBP stimulates transcription. EMBO J 17:2886-2893.

Roberson ED, Sweatt JD (1999) A biochemical blueprint for long-term memory. Learn Mem 6:381-388.

Roberson ED, English JD, Adams JP, Selcher JC, Kondratick C, Sweatt JD (1999) The mitogen-activated protein kinase cascade couples PKA and PKC to cAMP response element binding protein phosphorylation in area CA1 of hippocampus. J Neurosci 19:4337-4348.

Schafe GE, Atkins CM, Swank MW, Bauer EP, Sweatt JD, LeDoux JE (2000) Activation of ERK/MAP kinase in the amygdala is required for memory consolidation of Pavlovian fear conditioning. J Neurosci 20:8177-8187.

Selcher JC, Atkins CM, Trzaskos JM, Paylor R, Sweatt JD (1999) A necessity for MAP kinase activation in mammalian spatial learning. Learn Mem 6:478-490.

Silva AJ, Kogan JH, Frankland PW, Kida S (1998) CREB and memory. Annu Rev Neurosci 21:127-148.

Spencer VA, Davie JR (1999) Role of covalent modifications of histones in regulating gene expression. Gene 240:1-12.

Stork O, Welzl H (1999) Memory formation and the regulation of gene expression. Cell Mol Life Sci 55:575-592.

Swank MW (2000a) Phosphorylation of MAP kinase and CREB in mouse cortex and amygdala during taste aversion learning. NeuroReport 11:1625-1630.

Swank MW (2000b) Pharmacological antagonism of tyrosine kinases and MAP kinase in brainstem blocks taste aversion learning in mice. Physiol Behav 69:499-503.

Thomson S, Mahadevan LC, Clayton AL (1999) MAP kinase-mediated signalling to nucleosomes and immediate-early gene induction. Semin Cell Dev Biol 10:205-214.

Watts AG (2000) Understanding the neural control of ingestive behaviors: helping to separate cause from effect with dehydration-associated anorexia. Horm Behav 37:261-283.

Yoshida M, Kijima M, Akita M, Beppu T (1990) Potent and specific inhibition of mammalian histone deacetylase both in vivo and in vitro by trichostatin A. J Biol Chem 265:17174-17179. 